\title{
Absence of time-reversal symmetry breaking in the noncentrosymmetric superconductor $\mathrm{Mo}_{3} \mathrm{Al}_{2} \mathrm{C}$
}

\author{
E. Bauer, ${ }^{1}$ C. Sekine, ${ }^{2}$ U. Sai, ${ }^{2}$ P. Rogl,${ }^{3}$ P. K. Biswas ${ }^{4}$ and A. Amato ${ }^{4}$ \\ ${ }^{1}$ Institute of Solid State Physics, Vienna University of Technology, A-1040 Wien, Austria \\ ${ }^{2}$ Muroran Institute of Technology, Muroran 050-8585, Japan \\ ${ }^{3}$ Institute of Physical Chemistry, University of Vienna, A-1090 Wien, Austria \\ ${ }^{4}$ Laboratory for Muon Spin Spectroscopy, Paul Scherrer Institute, CH-5232 Villigen PSI, Switzerland \\ (Received 1 April 2014; revised manuscript received 7 August 2014; published 27 August 2014)
}

\begin{abstract}
Zero-field muon spin rotation and relaxation $(\mu \mathrm{SR})$ studies carried out on the strongly coupled, noncentrosymmetric superconductor $\mathrm{Mo}_{3} \mathrm{Al}_{2} \mathrm{C}, T_{c}=9 \mathrm{~K}$, did not reveal hints of time-reversal symmetry breaking as was found for a number of other noncentrosymmetric systems. Transverse field measurements performed above and below the superconducting transition temperature defined the temperature dependent London penetration depth, which in turn served to derive from a microscopic point of view a simple $s$-wave superconducting state in $\mathrm{Mo}_{3} \mathrm{Al}_{2} \mathrm{C}$. The present investigations also provide fairly solid grounds to conclude that time-reversal symmetry breaking is not an immanent feature of noncentrosymmetric superconductors.
\end{abstract}

DOI: 10.1103/PhysRevB.90.054522

PACS number(s): 74.25.Bt, 74.70.Ad, 72.15.Eb

\section{INTRODUCTION}

The search for superconductors in the previous decades was either devoted to find high transition temperatures, or to explore unconventional superconducting states which in different aspects deviate from the expectations of the BCS model. For the latter, superconducting states beyond $s$-wave superconductivity, i.e, $p-, d$-, or $f$-wave states, are of primary interest, where either spin-triplet pairing occurs, or orbital contributions $l>0$ are involved. Superconductivity with $l>0$ is maintained by spin fluctuations rather than by phonons as it is most likely the case, e.g., in the group of heavy fermion superconductors (see, e.g., Refs. [1,2]).

Unconventional behavior was recently found in some members of the family of noncentrosymmetric (NCS) superconductors, characterized by the absence of inversion symmetry in the respective crystal structure. NCS superconductors like $\mathrm{CePt}_{3} \mathrm{Si}$ [3] (for a recent review see e.g., Ref. [4]) have evidenced a mixing of spin singlet and spin triplet Cooper pairs in the superconducting condensate, although it was pointed out by Anderson [5] that spin-triplet pairing requires a center of inversion in the crystal structure as an essential symmetry element. Time-reversal symmetry (TRS), on the other hand, provides the necessary conditions for spin-singlet Cooper pairing [6].

Since compounds like $\mathrm{CePt}_{3} \mathrm{Si}$ exhibit beside the NCS crystal structure strong correlations among electrons, both conditions (i.e., the correlation strength and the absence of inversion symmetry in the crystal structure) simultaneously apply, forming a joint set of physical properties and phenomena. We aim to clarify whether or not unconventional superconductivity as found for $\mathrm{CePt}_{3} \mathrm{Si}$ results from the combination of strong electron correlations and missing of inversion symmetry or whether these properties are an exclusive result of the NCS crystal. For this, we have established a detailed research program, to check physical properties and the superconducting state of materials without strong correlations among electrons.

A relatively large number of NCS superconductors can be found in the class of ternary $E p T X_{3}$, where $E_{p}$ are electropositive elements like $\mathrm{Ba}$ or $\mathrm{Sr}, T$ are transition elements like $\mathrm{Ni}, \mathrm{Pd}$, or $\mathrm{Pt}$, and $X$ denotes $\mathrm{Si}$ or Ge. An element-dependent tuning of the antisymmetric spin-orbit coupling (ASOC), which lifts the twofold spin-degenerate state of the electronic bands, and which is the result of the absence of inversion symmetry [7], has been found from electronic structure calculations [8-10]. However, even if the band-splitting is large in EpT $X_{3}$ compounds, as it is the case for heavy elements like platinum, superconductivity appears to be a fully gapped $s$-wave BCS-like state.

Previously we have identified $\mathrm{Mo}_{3} \mathrm{Al}_{2} \mathrm{C}$ as an NCS superconductivity without significant correlations among electrons [11] (see also Ref. [12]). Electrical resistivity, specific heat, and nuclear magnetic resonance (NMR) measurements classified $\mathrm{NCS} \mathrm{Mo}_{3} \mathrm{Al}_{2} \mathrm{C}\left(\beta\right.$-Mn type, space group $\left.P 4_{1} 32\right)$ as a strongly-coupled superconductor with $T_{c}=9 \mathrm{~K}$. Besides, several properties observed from this study could not be explained in terms of a simple BCS superconductor. Thus, the possibility of an unconventional superconducting state was concluded. A London penetration depth study which we have carried out on $\mathrm{Mo}_{3} \mathrm{Al}_{2} \mathrm{C}$, indicated, however, a rather conventional behavior [13].

To reveal direct information about the superconducting state from microscopic probes, we have undertaken temperature dependent zero-field (ZF) and transverse-field (TF) muon spin rotation and relaxation $(\mu \mathrm{SR})$ studies at PSI.

$\mathrm{ZF}-\mu \mathrm{SR}$ is a very sensitive probe to detect small magnetic fields. This technique has been used extensively in the past to check for TRS broken superconducting states. The tiny spontaneous magnetic fields, which appear with the onset of superconductivity in these TRS breaking materials, result from the nonzero Cooper-pair moments which are locally aligned.

Right now, only few examples of superconductors that break time-reversal symmetry are known. Among them is the spin-triplet superconductor $\mathrm{Sr}_{2} \mathrm{RuO}_{4}$ [14], the heavy fermion materials $\mathrm{UPt}_{3}$ and $(\mathrm{U}, \mathrm{Th}) \mathrm{Be}_{13}[15,16]$, and some superconducting skutterudites [17-19]. Recently, however, NCS superconductors have been added to this group: $\mathrm{LaNiC}_{2}$ [20], $\mathrm{Re}_{6} \mathrm{Zr}$ [21], or the locally NCS system SrPtAs [22]. In most of the cases, $\mu \mathrm{SR}$ spectroscopy has been used to arrive at this conclusion. A recent $\mu \mathrm{SR}$ study concerning NCS BaPtSi${ }_{3}$ [23], however, did not reveal any measurable signal which would indicate TRS breaking in this system. 


\section{EXPERIMENT}

Polycrystalline samples were prepared at high temperatures and high pressures using a wedge-type cubic anvil high-pressure apparatus. The compounds were prepared by reacting stoichiometric amounts of $3 \mathrm{~N}$ (99.9\% pure) Mo, $3 \mathrm{~N}-\mathrm{Al}$, and $5 \mathrm{~N}-\mathrm{C}$ powders at $4 \mathrm{GPa}$. The reaction temperature was about $1200^{\circ} \mathrm{C}$. The prepared samples were characterized by powder x-ray diffraction using Co $K_{\alpha 1}$ radiation and silicon as a standard.

Magnetic susceptibility was measured with a Quantum Design MPMS superconducting quantum interference device magnetometer. The specific heat was measured by thermal relaxation method (PPMS, Quantum Design).

$\mu \mathrm{SR}$ measurements were performed at the Paul Scherrer Institute (PSI), Villigen, Switzerland. Zero-field (ZF) and transverse-field (TF) $\mu \mathrm{SR}$ experiments were carried out on the spectrometer DOLLY located at the beamline $\pi \mathrm{E} 1$ at PSI. About 10 disk-like samples (diameter: $1 \mathrm{~mm}$, height: $0.5 \mathrm{~mm}$ ) in the form of pressed pellets were mounted on a copper fork-shaped sample holder.

The sample was cooled from above $T_{c}$ to base temperature at $H=0$ during $\mathrm{ZF}-\mu \mathrm{SR}$ measurements and in a field of 150 Oe for TF- $\mu$ SR measurements. The typical counting statistics were $\approx 20$ million muon decays per data point. The ZF- and $\mathrm{TF}-\mu$ data were analyzed using the free software package MUSRFIT [24].

\section{RESULTS AND DISCUSSION}

In order to precharacterize the samples prepared by the high-pressure technique, heat capacity and magnetic susceptibility measurements have been carried out in a relevant temperature range, above and below the superconducting phase transition temperature. Shown in Fig. 1 is the temperature dependent specific heat, $C_{p}$, plotted as $C_{p} / T$ vs $T$ (left axis) and zero-field cooled magnetic susceptibility $\chi$, plotted as $\chi$

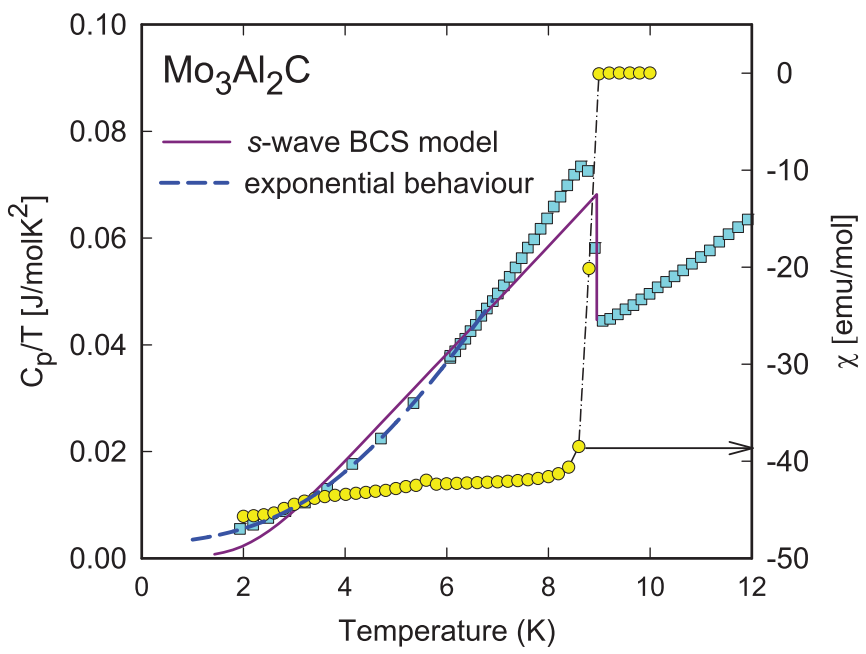

FIG. 1. (Color online) Low temperature behavior of the heat capacity $C_{p}$ of $\mathrm{Mo}_{3} \mathrm{Al}_{2} \mathrm{C}$ plotted as $C_{p} / T$ vs $T$ (referring to the left axis) and of the magnetic susceptibility $\chi$ (zero-field cooling, right axis). The solid line comprises the heat capacity of an $s$-wave superconductor in the weak coupling regime. The dashed line is a fit as explained in the text. vs $T$ (right axis). Obviously, both measurements reveal bulk superconductivity of $\mathrm{Mo}_{3} \mathrm{Al}_{2} \mathrm{C}$ at about $9 \mathrm{~K}$, in fine agreement with data reported earlier [11].

From a standard analysis of the heat capacity data in the normal state region, i.e., $C_{p}(T)=\gamma T+\beta T^{3}$, with $\gamma$ being the Sommerfeld coefficient and $\beta$ is proportional to the Debye temperature $\theta_{D}$, a moderately large $\gamma=17 \mathrm{~mJ} / \mathrm{mol} \mathrm{K}^{2}$ and $\theta_{D}=321 \mathrm{~K}$ is revealed, in perfect agreement with our previous results from a sample prepared on a different route [11].

The temperature dependent heat capacity exhibits a jump at $T=T_{c}$ of about $0.29 \mathrm{~J} / \mathrm{mol} \mathrm{K}$, revealing $\delta C / \gamma T_{c} \approx 1.94$, a value which is well above the BCS figures of 1.43 for this quantity. In general, such a behavior would be accounted for in terms of strong coupling superconductivity. Strong coupling is supported as well by an approximated formula of the specific heat in the superconducting state of an $s$-wave superconductor, i.e., $C_{e, s} \propto T^{1 / 2} \exp \left(-\Delta_{0} / k_{B} T\right)$, where the gap $\Delta_{0} \equiv \Delta(0)$, as derived from a least squares fit at low temperatures, arrives at $\Delta_{0} / k_{B}=18.3 \mathrm{~K}$ (compare the dashed line in Fig. 1). As a result, $2 \Delta_{0} / k_{B} T_{c}=4.06$, which is well above the standard weak-coupling BSC value $2 \Delta_{0} / k_{B} T_{c}=3.52$. The solid line sketches the behavior of an $s$-wave BCS superconductor in the weak coupling limit [25].

The above described and characterized samples were used to check the assumption whether or not TRS breaking is a property of a NCS superconductor and, additionally, to experimentally determine the London penetration depth, which as microscopic property can serve to reveal the superconducting state of $\mathrm{Mo}_{3} \mathrm{Al}_{2} \mathrm{C}$.

Figure 2 shows the $\mathrm{ZF}-\mu \mathrm{SR}$ spectra measured at $T=1.7$ and $15 \mathrm{~K}$, well below and above $T_{c}$. The time dependent asymmetry above and below $T_{c}$ does not show any distinct difference. This implies that there is no additional relaxation of the $\mu \mathrm{SR}$ signal appearing in the superconducting state of $\mathrm{Mo}_{3} \mathrm{Al}_{2} \mathrm{C}$, which therefore confirms that there are no spontaneous coherent internal magnetic fields associated, e.g., with long range magnetic order in $\mathrm{Mo}_{3} \mathrm{Al}_{2} \mathrm{C}$. The temperature dependence of the muon depolarization rate can be derived from an analysis of the present data in terms of the static Kubo-Toyabe function [26] together with a nondecaying

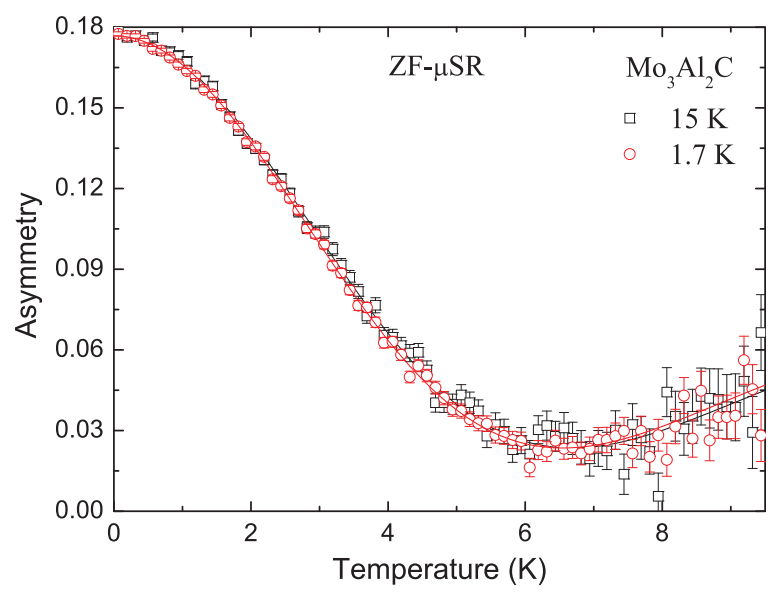

FIG. 2. (Color online) $\mu \mathrm{SR}$ zero field spectra of $\mathrm{Mo}_{3} \mathrm{Al}_{2} \mathrm{C}$ measured at $T=1.7$ and $15 \mathrm{~K}$, respectively. The solid lines are least squares fits according to the static Kubo-Toyabe formula, Eq. (1). 


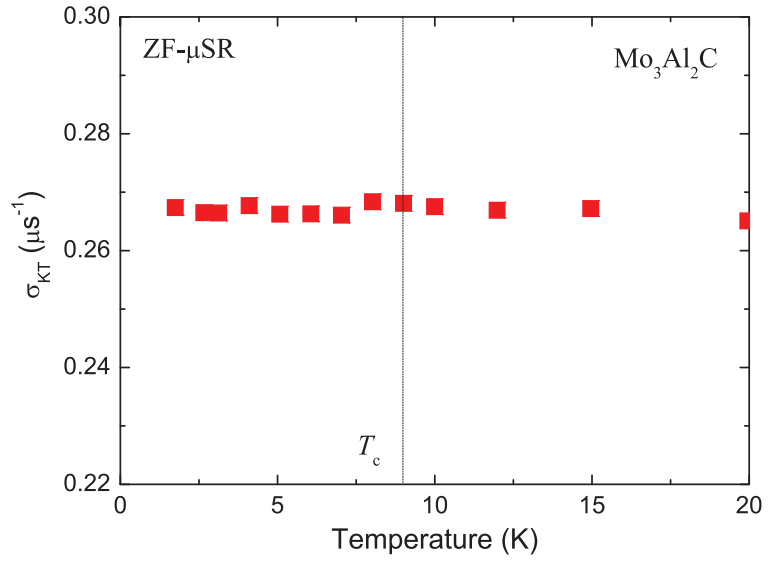

FIG. 3. (Color online) Zero-field muon depolarization rate $\sigma_{K T}$ of $\mathrm{Mo}_{3} \mathrm{Al}_{2} \mathrm{C}$.

constant background, $A_{b g d}$,

$$
A(t)=A(0)\left[\frac{1}{3}+\frac{2}{3}\left(1-\sigma_{K T}^{2} t^{2}\right) \exp \left(-\frac{\sigma_{K T}^{2} t^{2}}{2}\right)\right]+A_{b g d} .
$$

$A(0)$ is the initial asymmetry and $\sigma_{K T}$ is the muon spin relaxation rate, referring to the nuclear dipole moments.

For temperatures below and above $T_{c}$ the relaxation rate $\sigma_{K T}$ does not change within the resolution of the instrument $\left(\Delta \sigma_{K T}<0.002 \mu \mathrm{s}^{-1}\right.$, compare Fig. 3). This indicates the absence of any spontaneous magnetic fields occurring in the superconducting state. Distinct changes at $T=T_{c}$, however, were found in archetypal $\mathrm{Sr}_{2} \mathrm{RuO}_{4}$ [14], in $\mathrm{LaNiGa}_{2}$ [27], or in NCS superconductors $\mathrm{LaNiC}_{2}$ [20], $\operatorname{Re}_{6} \mathrm{Zr}$ [21], or in the locally NCS SrPtAs [22]. Just to mention a few examples, the change in $\sigma_{K T}$ ranges from about $0.01 \mu \mathrm{s}^{-1}\left(\mathrm{LaNiGa}_{2}\right.$ [27]) to about $0.04 \mu \mathrm{s}^{-1}\left(\mathrm{Sr}_{2} \mathrm{RuO}_{4}\right.$ [14]), a magnitude of a signal which easily should also be resolvable from the present experiment. For the former compounds, TRS breaking was concluded; it was independently confirmed by Kerr effect measurements in the case of $\mathrm{Sr}_{2} \mathrm{RuO}_{4}$ [28]. Thus it can be argued on solid ground that the present $\mu \mathrm{SR}$ study does not reveal any hint for TRS breaking in $\mathrm{Mo}_{3} \mathrm{Al}_{2} \mathrm{C}$. There are further examples of NCS superconductors exhibiting no TRS such as $\mathrm{BaPtSi}_{3}$ [23] or $\mathrm{LaPt}_{3} \mathrm{Si}$ [29]. From these observations one may safely conclude that TRS is not an inherent property of NCS superconductors.

To derive a microscopic view of the superconducting state in $\mathrm{Mo}_{3} \mathrm{Al}_{2} \mathrm{C}$, in particular with respect to the gap-structure, transverse field (TF) $\mu$ SR measurements have been carried out for temperatures below and above $T_{c}$ in a transverse field of 150 Oe. A set of typical measurements (below and above $T_{c}$ ) is shown in Figs. 4(a) and 4(b). Obviously, there are significant differences between both measurements, characterized by a very large depolarization rate in the superconducting state. In order to quantitatively analyze the experimental data, the following oscillatory decaying Gaussian function was employed:

$$
\begin{aligned}
A^{T F}(t)= & A^{T F}(0) \exp \left(-\frac{\sigma^{2} t^{2}}{2}\right) \cos \left(\gamma_{\mu} B_{\text {int }} t+\phi\right) \\
& +A_{b g d}^{T F}(0) \cos \left(\gamma_{\mu} B_{b g d} t+\phi\right) .
\end{aligned}
$$
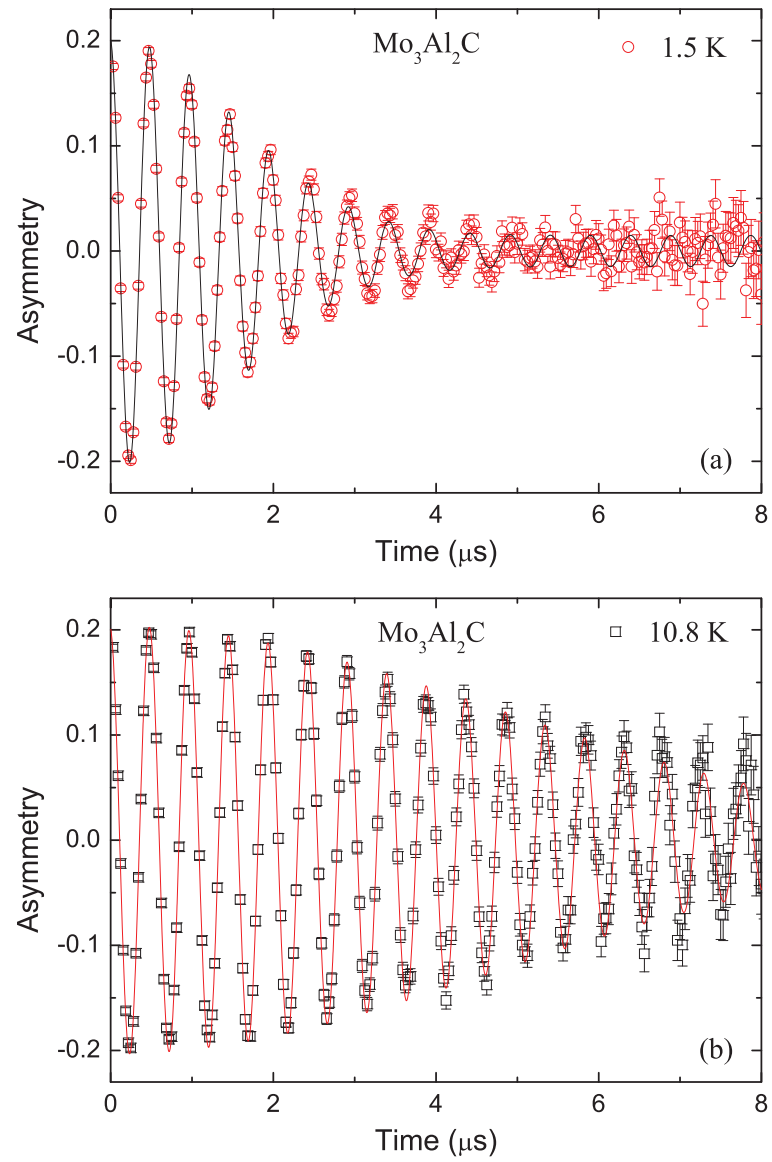

FIG. 4. (Color online) $\mu \mathrm{SR}$ transverse field spectra of $\mathrm{Mo}_{3} \mathrm{Al}_{2} \mathrm{C}$ at external magnetic fields $\mu_{0} H=150$ Oe for temperatures below (a) and above (b) the superconducting phase transition $T_{c}=9 \mathrm{~K}$. The solid lines are least squares fits according to Eq. (2).

$A^{T F}(0)$ is the initial asymmetry, $\gamma_{\mu} / 2 \pi=135.5 \mathrm{MHz} / \mathrm{T}$ is the muon gyromagnetic ratio, and $B_{\text {int }}$ and $B_{b g d}$ are the internal and the background magnetic fields at the muon sites, respectively. The initial phase offset is given by $\phi$, and $\sigma$ is the Gaussian muon spin relaxation rate with $\sigma=\left(\sigma_{s c}^{2}+\sigma_{n m}^{2}\right)^{1 / 2} . \sigma_{s c}$ is the superconducting contribution to the relaxation rate due to the field variation across the flux line lattice and $\sigma_{n m}$ is the nuclear magnetic dipolar contribution, assumed to be constant in the temperature range considered here.

Least squares fits according to Eq. (2) to data above and below $T_{c}$ are shown in Fig. 4 as solid lines. A summary of the Gaussian muon spin relaxation rate $\sigma$ is displayed in Fig. 5. The temperature dependent variation of $\sigma$ exhibits a pronounced change at $T=T_{c}$ and in the superconducting state a mean-field-like behavior is deduced. Subtracting the normal state contribution from the data derived $\left(\sigma_{n m}=0.2 \mu \mathrm{s}^{-1}\right)$ reveals the superconducting contribution $\sigma_{s c}$. The later is related to the penetration depth $\lambda$ and thus to the superfluid density $n_{s}$.

In terms of a Ginzburg-Landau treatment of the vortex state, Brandt [30] has shown that the penetration depth can be evaluated from $\sigma_{s c}$, if the temperature dependent upper 


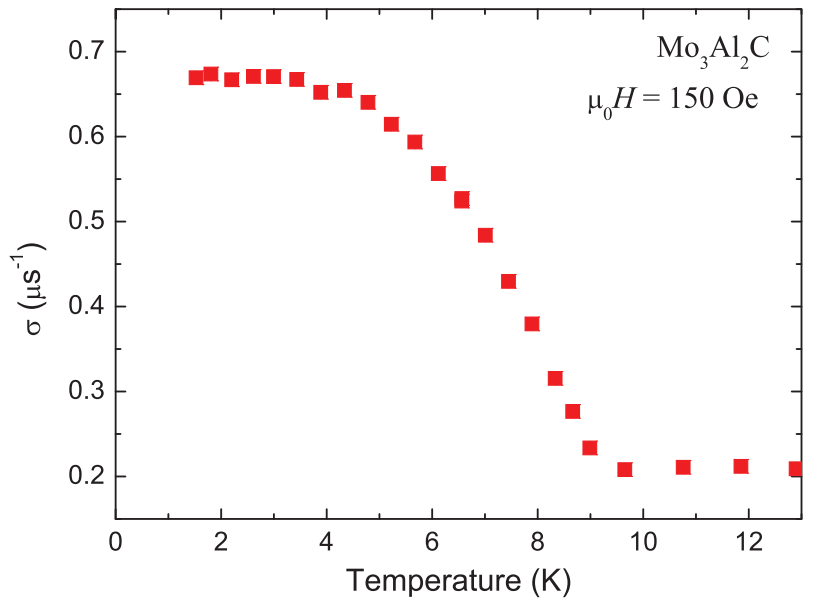

FIG. 5. (Color online) Temperature dependent muon spin relaxation rate $\sigma$ of $\mathrm{Mo}_{3} \mathrm{Al}_{2} \mathrm{C}$.

critical field, $\mu_{o} H_{c 2}$, is known, i.e.,

$$
\begin{aligned}
\sigma_{s c}= & 4.83 \times 10^{4}\left(1-\frac{\mu_{0} H}{\mu_{0} H_{c 2}}\right) \\
& \times\left(1+1.21\left(1-\frac{\mu_{0} H}{\mu_{0} H_{c 2}}\right)^{0.5}\right)^{3} \lambda^{-2} .
\end{aligned}
$$

Equation (3) is valid for $\kappa=\lambda / \xi>5\left[\kappa\left(\mathrm{Mo}_{3} \mathrm{Al}_{2} \mathrm{C}\right) \approx 76\right]$. The upper critical field was already observed in Ref. [11]. Combining the data of the upper critical magnetic field of $\mathrm{Mo}_{3} \mathrm{Al}_{2} \mathrm{C}$ with the superconducting contribution to the relaxation rate, $\sigma_{s c}$, derived from the present experiment, allows us to obtain the temperature dependent relaxation rate $\lambda(T)$ employing Eq. (3). Results of this procedure are displayed in Fig. 6. Upon an increase of temperature, the penetration depth of $\lambda(T)$ reduces continuously and vanishes at $T=T_{c}$. The temperature dependence of $\lambda$ provides a possibility to study the order parameter of a certain superconductor on a microscopic level, i.e., the gap in the electronic density

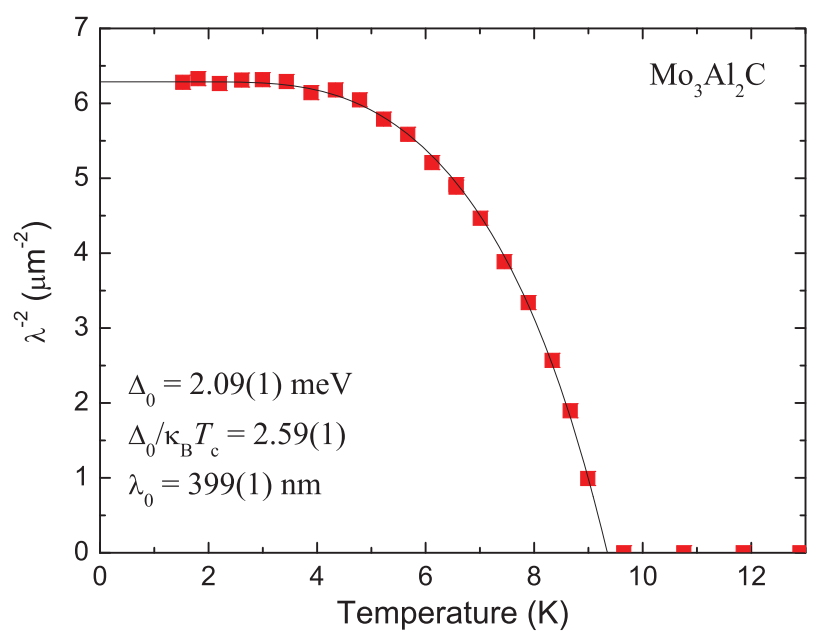

FIG. 6. (Color online) Temperature dependent penetration depth $\lambda$ of $\mathrm{Mo}_{3} \mathrm{Al}_{2} \mathrm{C}$ plotted as $\lambda^{-2}$ vs $T$. The solid line is a least-squares fit according to an $s$-wave BCS model. of states in the proximity of the Fermi energy. Assuming $s$-wave superconductivity with a single gap $\Delta$ for $\mathrm{Mo}_{3} \mathrm{Al}_{2} \mathrm{C}$ reveals [31]

$$
\frac{\lambda^{-2}(T, \Delta(0))}{\lambda^{-2}(0, \Delta(0))}=1+2 \int_{\Delta(0)}^{\infty} \frac{\partial f}{\partial E} \frac{E d E}{\sqrt{E^{2}-\Delta(T)^{2}}},
$$

with $f=\left[1+\exp \left(E / k_{B} T\right]^{-1}\right.$ being the Fermi-Dirac distribution function. The gap $\Delta(T)$ is assumed to follow the standard interpolation function $\Delta(T)=$ $\Delta(0) \tanh \left[\left[\pi k_{B} T_{c} / \Delta(0)\right] \sqrt{a\left[\left(T_{c} / T\right)-1\right]}\right]$ [32]. The constant $a$ depends on the coupling strength and the geometry of the gap.

Applying Eq. (4) to the experimental data of Fig. 6 reveals a convincing fit (solid line, Fig. 6) for $\Delta_{0}=2.09(1)$ $\mathrm{meV}, \Delta_{0} / k_{B} T_{c}=2.59(1)$, and $\lambda(0)=399(80) \mathrm{nm}$. Excellent agreement is obtained, when comparing the penetration depth at $T=0$ obtained from the present microscopic study, to this quantity derived from an analysis of bulk properties in $\mathrm{Mo}_{3} \mathrm{Al}_{2} \mathrm{C}$ [11], $\lambda^{\text {bulk }}(0)=380 \mathrm{~nm}$. Accurate simulations by Sonier et al., [33], have shown that under reasonable assumptions of disorder, typical errors of the penetration depth, $\lambda$, are at a level of $20 \%$. Considering statistical and systematic errors in this study, the penetration depth of $\mathrm{Mo}_{3} \mathrm{Al}_{2} \mathrm{C}$ can be evaluated with an error bar of about $80 \mathrm{~nm}$. Furthermore, the perfect agreement of an $s$-wave BCS model with the present temperature dependent penetration depth supports a nodeless superconducting state below $T_{c}=9 \mathrm{~K}$ in $\mathrm{Mo}_{3} \mathrm{Al}_{2} \mathrm{C}$. Strong coupled superconductivity, however, can be argued from $\Delta_{0} / k_{B} T_{c}=2.59(1)$, in convincing agreement with the jump of the heat capacity at $T=T_{c}$ and the exponential behavior of $C_{e s}$ at low temperatures revealing $2 \Delta_{0} / k_{B} T_{c}=4.06 \mathrm{meV}$.

\section{SUMMARY}

Specific heat, magnetic susceptibility and $\mu$ SR studies have been performed on ternary $\mathrm{Mo}_{3} \mathrm{Al}_{2} \mathrm{C}$, revealing strong coupling superconductivity below $T_{c}=9 \mathrm{~K}$. Muons as microscopic probes have been used to retrieve information regarding the possibility of time reversal symmetry breaking, which could be a natural consequence of the mixing of spin singlet and spin triplet states in the superconducting condensate. The latter results from the asymmetric spin-orbit coupling as a consequence of the missing of inversion symmetry in the crystal structure of $\mathrm{Mo}_{3} \mathrm{Al}_{2} \mathrm{C}$. Experimental results, however, do not evidence TRS breaking, at least within the resolution of the experimental facility. Since microscopic studies on other NCS superconductors like $\mathrm{BaPtSi}_{3}$ or $\mathrm{LaPt}_{3} \mathrm{Si}$ have not shown this phenomenon, it is rather likely that TRS breaking cannot be considered as a further immanent feature of NCS superconductors. The results of this research form another development in our aim to define conditions and consequences of superconductivity in materials with missing inversion symmetry: TRS breaking is not an $a$ priori condition either in weakly or in strongly coupled NCS superconductors.

Since the muon spin relaxation rate is inversely proportional to the London penetration depth $\lambda$, an accurate temperature 
dependence of $\lambda(T)$ was derived in this study. Based on $s$ wave superconductivity and a single gap $\Delta, \lambda(T)$ was perfectly modeled, revealing substantiated hints for strongly coupled superconductivity in $\mathrm{Mo}_{3} \mathrm{Al}_{2} \mathrm{C}$ and well founded evidence of a nodeless state of the superconducting order parameter.

\section{ACKNOWLEDGMENTS}

This research was supported by the Austrian Science Fund (FWF) Project No. P22290 and a Grant-in-Aid for Scientific Research (B) (No. 23340092) of the Japan Society for the Promotion of Science.
[1] G. Goll, Unconventional Superconductors - Experimental Investigations of the Order-Parameter Symmetry (Springer-Verlag, New York, 2009).

[2] Ch. Pfleiderer, Rev. Mod. Phys. 81, 1551 (2009).

[3] E. Bauer, G. Hilscher, H. Michor, C. Paul, E. W. Scheidt, A. Gribanov, Y. Seropegin, H. Noël, M. Sigrist, and P. Rogl, Phys. Rev. Lett. 92, 027003 (2004).

[4] E. Bauer and M. Sigrist, Non-Centrosymmetric Superconductors: Introduction and Overview, Lecture Notes in Physics (Springer-Verlag, Berlin, 2012).

[5] P. W. Anderson, Phys. Rev. B 30, 4000 (1984).

[6] P. W. Anderson, J. Phys. Chem. Solids 11, 26 (1959).

[7] L. P. Gor'kov and E. I. Rashba, Phys. Rev. Lett. 87, 037004 (2001).

[8] E. Bauer, R. T. Khan, H. Michor, E. Royanian, A. Grytsiv, N. Melnychenko-Koblyuk, P. Rogl, D. Reith, R. Podloucky, E.-W. Scheidt, W. Wolf, and M. Marsman, Phys. Rev. B 80, 064504 (2009).

[9] F. Kneidinger, Thesis, Vienna University of Technology, 2014.

[10] F. Kneidinger, L. Salamakha, E. Bauer, I. Zeiringer, P. Rogl, C. Blaas-Schenner, D. Reith, and R. Podloucky, Phys. Rev. B 90, 024504 (2014).

[11] E. Bauer, G. Rogl, X.-Q. Chen, R. T. Khan, H. Michor, G. Hilscher, E. Royanian, K. Kumagai, D. Z. Li, Y. Y. Li, R. Podloucky, and P. Rogl, Phys. Rev. B 82, 064511 (2010).

[12] A. B. Karki, Y. M. Xiong, I. Vekhter, D. Browne, P. W. Adams, D. P. Young, K. R. Thomas, Julia Y. Chan, H. Kim, and R. Prozorov, Phys. Rev. B 82, 064512 (2010).

[13] I. Bonalde, H. Kim, R. Prozorov, C. Rojas, P. Rogl, and E. Bauer, Phys. Rev. B 84, 134506 (2011).

[14] G. M. Luke, Y. Fudamoto, K. M. Kojima, M. I. Larkin, J. Merrin, B. Nachumi, Y. J. Uemura, Y. Maeno, Z. Q. Mao, Y. Mori et al., Nature (London) 394, 558 (1998).

[15] G. M. Luke, A. Keren, L. P. Le, W. D. Wu, Y. J. Uemura, D. A. Bonn, L. Taillefer, and J. D. Garrett, Phys. Rev. Lett. 71, 1466 (1993).

[16] R. H. Heffner, J. L. Smith, J. O. Willis, P. Birrer, C. Baines, F. N. Gygax, B. Hitti, E. Lippelt, H. R. Ott, A. Schenck et al., Phys. Rev. Lett. 65, 2816 (1990).

[17] Y. Aoki, A. Tsuchiya, T. Kanayama, S. R. Saha, H. Sugawara, H. Sato, W. Higemoto, A. Koda, K.
Ohishi, K. Nishiyama et al., Phys. Rev. Lett. 91, 067003 (2003).

[18] L. Shu, W. H. Y. Aoki, A. D. Hillier, K. Ohishi, K. Ishida, R. Kadono, A. Koda, O. O. Bernal, D. E. MacLaughlin, Y. Tunashima et al., Phys. Rev. B 83, 100504(R) (2011).

[19] A. Maisuradze, W. Schnelle, R. Khasanov, R. Gumeniuk, M. Nicklas, H. Rosner, A. Leithe-Jasper, Y. Grin, A. Amato, and P. Thalmeier, Phys. Rev. B 82, 024524 (2010).

[20] A. D. Hillier, J. Quintanilla, and R. Cywinski, Phys. Rev. Lett. 102, 117007 (2009).

[21] R. P. Singh, A. D. Hillier, B. Mazidian, J. Quintanilla, J. F. Annett, D. M. Paul, G. Balakrishnan, and M. R. Lees, Phys. Rev. Lett. 112, 107002 (2014).

[22] P. K. Biswas, H. Luetkens, T. Neupert, T. Stürzer, C. Baines, G. Pascua, A. P. Schnyder, M. H. Fischer, J. Goryo, M. R. Lees, H. Maeter, F. Brückner, H.-H. Klauss, M. Nicklas, P. J. Baker, A. D. Hillier, M. Sigrist, A. Amato, and D. Johrendt, Phys. Rev. B 87, 180503(R) (2013).

[23] K. Miliyanchuk, F. Kneidinger, C. Blaas-Schenner, D. Reith, R. Podloucky, P. Rogl, T. Khan, L. Salamakha, G. Hilscher, H. Michor, E. Bauer, and A. D. Hillier, J. Phys.: Conf. Ser. 273, 012078 (2011).

[24] A. Suter and B. M. Wojek, Phys. Procedia 30, 69 (2012).

[25] B. Mühlschlegel, Z. Phys. 155, 313 (1959).

[26] R. Kubo, Hyperfine Interact. 8, 731 (1981).

[27] A. D. Hillier, J. Quintanilla, B. Mazidian, J. F. Annett, and R. Cywinski, Phys. Rev. Lett. 109, 097001 (2012).

[28] J. Xia, Y. Maeno, P. T. Beyersdorf, M. M. Fejer, and A. Kapitulnik, Phys. Rev. Lett. 97, 167002 (2006).

[29] I. Kawasaki, I. Watanabe, H. Amitsuka, K. Kunimori, H. Tanida, and Y. Onuki, J. Phys. Soc. Jpn. 82, 084713 (2013).

[30] E. H. Brandt, Phys. Rev. B 68, 054506 (2003).

[31] M. Tinkham, Introduction to Superconductivity, 2nd ed. (McGraw-Hill, New York, 1996).

[32] F. Gross, B. S. Chandrasekhar, D. Einzel, K. Andres, P. J. Hirschfeld, H. R. Ott, J. Beuers, Z. Fisk, and J. L. Smith, Z. Phys. B 64, 175 (1986).

[33] J. E. Sonier, W. Huang, C. V. Kaiser, C. Cochrane, V. Pacradouni, S. A. Sabok-Sayr, M. D. Lumsden, B. C. Sales, M. A. McGuire, A. S. Sefat, and D. Mandrus, Phys. Rev. Lett. 106, 127002 (2011). 